\title{
Liquid crystal epoxy resins based on biphenyl group cured with aromatic amines - studied by dielectric spectroscopy
}

\author{
M. Wlodarska • A. Maj • B. Mossety-Leszczak • \\ G. W. Bak • H. Galina $\cdot$ L. Okrasa • M. Izdebski
}

Received: 19 April 2013 / Accepted: 17 July 2013 /Published online: 7 August 2013

(C) The Author(s) 2013. This article is published with open access at Springerlink.com

\begin{abstract}
In this paper we investigate the dielectric response of cured epoxy resins based on biphenyl group. We chose two monomers, which were earlier synthesised, containing the same mesogen but with long and short symmetric tails. They were cured with standard amines (DDM, DMAP). Firstly we optimised conditions of the curing process by dielectric and optical observation in situ. Small differences in the dynamics of the curing process were observed in different mixtures. For the four selected products we made dielectric measurements in a wide range of frequency and temperature. Although, generally, dielectric response is similar in all the cured materials, differences can be seen in the details. At lower temperatures, quite strong relaxation process is seen in all products, its low activation energy points towards a $\beta$-process, which appears in many polymers. The Havriliak-Negami formula was fitted to the data and characteristic parameters of the observed relaxation processes were compared in detail. In some cases the $\alpha$-process also appears. We conclude how the longer spacer and the type of curing process influence the electrical properties of the resin.
\end{abstract}

Keywords Dielectric spectroscopy $\cdot$ Liquid crystal polymer networks · Epoxy resins

M. Wlodarska $(\bowtie) \cdot$ A. Maj $\cdot$ G. W. Bak $\cdot$ M. Izdebski

Institute of Physics, Technical University of Lodz, Wolczanska 219, 90-924 Lodz, Poland

e-mail: magdaw@p.lodz.pl

B. Mossety-Leszczak $\cdot$ H. Galina

Department of Industrial and Materials Chemistry, Rzeszow

University of Technology, W. Pola 2, 35-959 Rzeszow, Poland

L. Okrasa

Department of Molecular Physics, Technical University of Lodz, Zeromskiego 116, 90-924 Lodz, Poland

\section{Introduction}

Nowadays, new technical developments create a demand for new materials, one of the examples is using liquid crystalline epoxy resins to produce polymer networks [1-10]. On the other hand, recent research has shown that properties of epoxy polymers can be improved by addition of some inorganic materials as fillers [11-15]. Liquid crystal epoxy networks may be used to produce oriented matrices - for example, to induce global orientation of nanoparticles $[6,7,16]$. In such networks, not only the structure but also the curing conditions are a little different than in the case of traditional resins [17, 18]. Generally, polymer networks are created as a result of curing which means formation of cross-linking bonds between neighboring molecules. The reaction rate depends on many factors such as type of the monomer and curing agent, temperature and duration of keeping the mixture at that temperature. The curing conditions affect the physical properties of the final products, e.g. the vitrification temperature [19-21].

Many experimental techniques exist which enable observation of the glassy transition. This stems from the fact that not only thermodynamic properties change abruptly during transition to the glassy state, but also a series of other properties [22, 23]. For instance, dynamic thermomechanical analysis or dielectric spectroscopy not only allow for determination of the vitrification temperature but can deliver rich information about relaxation processes occurring in the studied material. The technique of dielectric spectroscopy is based on measuring changes in the dielectric properties of the sample in response to the applied alternating electric field. The reaction of sample polarization to a change in the electric field is delayed, which can be observed as a relaxation process with characteristic relaxation time. In polymers, two or three relaxation processes are most often observed, which are denoted with greek letters $\alpha, \beta, \gamma[24-30]$. The (structural) $\alpha$-process usually corresponds to the glass transition. It is thereby related to hindering (freezing) of the motion of polymer chain segments, 
caused by approaching the vitrification temperature Tg. The $\alpha-$ process is among the slowest ones in polymers, the processes appearing at higher frequencies are customarily described as $\beta$-, $\gamma$-processes. At present, it is believed that $\beta$-process reflects rotational motions of polar group fragments containing single bonds, inside the molecule [26-28]. These motions can be treated as more or less free rotations of dipolar groups. The $\alpha-$ and $\beta$-processes exhibit different temperature dependencies. The relaxation time of the process $(\beta, \gamma)$ can usually be described with the Arrhenius formula [26-30]:

$\frac{1}{\tau}=\frac{1}{\tau_{0}} \exp \left(-\frac{E_{a}}{R T}\right) ;$

However, abrupt hindering of the dynamics near the vitrification temperature results in a deviation from that exponential dependency. To describe dynamic properties of glass - e.g. the structural $(\alpha)$ relaxation time - an empirical formula proposed by Vogel, Tammann and Fulcher (VTF) is used [26-30]:

$\tau=\tau_{0} \exp \left(\frac{B}{k\left(T-T_{0}\right)}\right)$

where $\mathrm{k}$ - Boltzmann constant; $\mathrm{B}, \mathrm{T}_{0}$ - constants characteristic for given material (for $\mathrm{T}_{0}=0$ the VTF equation transforms into the Arrhenius formula). Vanishing of the structural process therefore determines the vitrification temperature. This technique delivers important data about phenomena occurring in the studied materials, also in the course of the curing process. Its big advantage is the possibility to carry out measurements in a wide range of frequency and temperature. In our work we performed dielectric measurements for two cured epoxy resins containing the biphenyl group. We used two different amines and compared the obtained results. The results of in situ dielectric observation during the curing process are also presented.

\section{Materials}

As precursors for the polymer networks we chose two epoxy monomers with biphenyl group and two aromatic amines DDM and DMAP. The molecular structure of the used materials is shown below (Fig. 1). The synthetic route and basic physical properties of the monomers were described elsewhere [31-33]. The presence of the biphenyl group caused the appearance of a smectic $\mathrm{B}$ phase in both the monomers in the temperature range of $90-114^{\circ} \mathrm{C}$ [31-33]. The selection of curing agents and curing conditions was preceded by optical observation. The mixture with DDM amine was prepared in stoichiometric ratio whereas in DMAP case the amine was only added as a catalyser (ca. $1 \%$ of the mixture). All the final products taken for further investigation were cured for $3 \mathrm{~h}$, the temperatures of curing are presented in Table 1.

\section{Experimental}

The optical observations of the mixtures were done using a polarizing microscope with crossing (and not crossing) polaroids at a magnification of $80 \times$, and a digital camera Moticam 2000. The material was introduced between plain glass plates.

The dielectric response during curing was recorded using Solartron 1260 Impedance Analyser with Chelsea Dielectric Interface 1295 . The real and imaginary components of capacitance were evaluated in the frequency range from $10^{-2}$ to $10^{5} \mathrm{~Hz}$. The temperature of the samples was stabilized with the accuracy of $0.1 \mathrm{~K}$ using Unipan 620 temperature controller. The diameter of parallel electrodes was $5 \mathrm{~mm}$ with a spacing of $50 \mu \mathrm{m}$. Dielectric measurements for all the products were performed in a wide range of frequency $\left(10^{6}-10^{-1} \mathrm{~Hz}\right)$ in the cooling route, using the Alpha high-resolution dielectric analyzer manufactured by Novocontrol. Data were controlled by a Quatro cryosystem (Novocontrol) and collected after thermal stabilization (with accuracy of $0.1 \mathrm{~K}$ ).
Fig. 1 Molecular structure of the used precursors (monomers and amines) and acronyms thereof

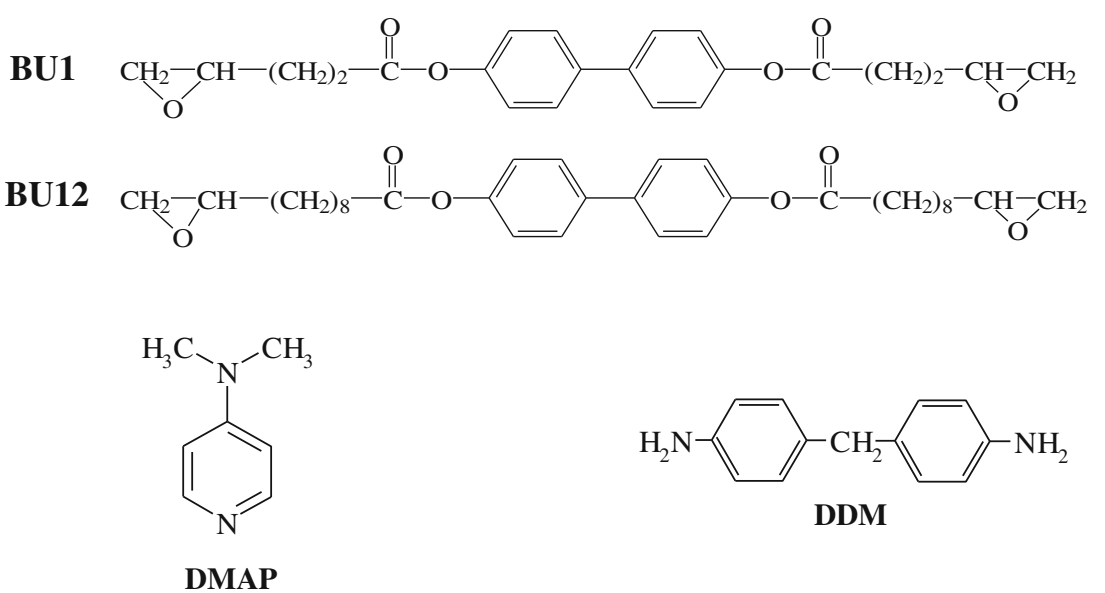


Table 1 Curing conditions of the investigated materials

\begin{tabular}{llll}
\hline Name & Monomer & Amine & $\mathrm{T}_{\text {cure }}\left[{ }^{\circ} \mathrm{C}\right]$ \\
\hline Product 1 & BU1 & DDM & 122 \\
Product 2 & BU12 & DDM & 138 \\
Product 3 & BU1 & DMAP & 110 \\
Product 4 & BU12 & DMAP & 127 \\
\hline
\end{tabular}

\section{Results and discussion}

Firstly, dielectric monitoring during isothermal cure was done for all the materials. Due to relatively high temperatures of curing which are accompanied in these materials by significant electric conductivity, the results were analyzed using the electric modulus $\left(M^{*}\right)$ representation. This quantity is related to the dielectric permittivity by the formula [34]:

$\varepsilon^{*}=\frac{1}{M^{*}}$

This approach allows observation of the reaction dynamics through changes occurring in the cured system which are well reflected in the plots of the electric modulus vs. time.

Figure 2a presents exemplary 3D plots for the second material (Table 1). A peak visible in the modulus representation in the observed frequency range is directly related with ionic conductivity. The constant $\tau_{\sigma}$ which corresponds to the frequency at which $M$ " reaches its maximum value is called the conductivity relaxation time and its value is given by the expression [34]:

$\tau_{\sigma}=\frac{\varepsilon_{0} \varepsilon_{\infty}}{\sigma_{0}}$

Plots of the $M$ " peak frequency as a function of time are shown for all the four products (Fig. 2b, c). Monitoring of the curing reaction was continued for $200 \mathrm{~min}$ and revealed a period of rapid, significant changes after which only slowly progressing, small changes were visible in all the cases. Some differences in the dynamics of the curing process were observed between different mixtures. In particular, when the monomers with long aliphatic chains were used (product 2 and 4), the conductivity peak was clearly changing during the first hour but then it stabilized and remained practically constant afterwards. In the other products the initial period of rapid changes is much shorter which means that the progress of the reaction slows down substantially after just several minutes, so longer curing time (or post-curing at a higher temperature) may be needed to obtain fully reacted product. The dynamics of the reaction is different for amines DDM and DMAP, in the first case the curing process starts without delay whereas in the second case the materials have to be kept at the curing temperature for a few minutes before the reaction gets initiated.

In the next step, dielectric studies were carried out for the obtained products (Table 1), in a wide range of frequency and temperature. To describe in details the relaxation processes in product 1 , the permittivity representation was chosen. However, only the lower temperatures where the peaks appear were taken into account - otherwise the data would be masked by conductivity (Fig. 3). In the permittivity representation, two peaks connected with relaxation processes are visible (figs. 3a, b). These processes were analyzed by fitting the Havriliak-Negami (HN) formula with conductivity term to the experimental data [35]:

$$
\varepsilon^{*}(\omega)-\varepsilon_{\infty}=-i \frac{\sigma_{0}}{\varepsilon_{0} \omega^{s}}+\sum_{k} \frac{\Delta \varepsilon_{k}}{\left(1+\left(i \omega \tau_{k}\right)^{\alpha_{k}}\right)^{\beta_{k}}}
$$

Here $\sigma_{0}$ is the DC conductivity, $\mathrm{s} \leq 1$ is a parameter describing for non-ohmic effects in the conductivity, $\varepsilon_{0}$ is the electric permittivity of vacuum, $\omega=2 \pi f$ is the radial frequency, $\varepsilon_{\infty}$ is $\varepsilon^{\prime}$ at $f \rightarrow \infty$. The dielectric strength $\Delta \varepsilon$ is the difference between the high-frequency and the low-frequency electric permittivity corresponding to the relaxation process under consideration, $\tau$ is a characteristic relaxation time, $\alpha$ and $\beta$ are shape parameters of the relaxation time spectra. Typical fitting results for the product 1 at two temperatures $303 \mathrm{~K}$ and $353 \mathrm{~K}$ - are shown in Fig. 3c. At each temperature, all the fitting parameters were calculated and an example of the fitting is shown in Fig. 3d. Both the relaxation processes are of strongly nonDebye type, with parameters $\alpha$ and $\beta$ not equal to one. The first process, appearing at the higher temperature, is similar to a structural $\alpha$-process and the second one is a $\beta$-process probably connected with molecular activity of dipolar groups (COO groups in the mesogen). According to the theoretical background, disappearing of the $\alpha$-process is connected with the temperature of glass transition, which could be estimates in this case at $333 \mathrm{~K}$. The calculated activation energy for the $\beta$-process $(E a=0.39 \mathrm{eV})$ is typical for this kind of processes.

A similar dielectric response was seen in product 2 (Fig. 4). The coexistence of two processes was shown in a wide range of temperature in modulus representation (Fig. 4a). The first process ( $\alpha$-process) was detected in a wide range of temperature and disappeared at $303 \mathrm{~K}$. This gives an estimate of the glass transition temperature, which is lower than in the previous material (Fig. 4c). The first $\alpha$-process is not as strong and well visible as in product 1 and below $343 \mathrm{~K}$ it exists together with the second process ( $\beta$-process). Typical observation data along with the fitting curves for both the processes were shown in Fig. 4b. The activation energy for the $\beta$-process is quite high $(E a=0.81 \mathrm{eV})$ and much higher than in product 1 . 
Fig. 2 Observations of the curing process through dielectric spectroscopy for all the investigated mixtures: a) an example of a $3 \mathrm{D}$ plot showing the imaginary part of the electric modulus vs. frequency and time for mixture 2 (monomer BU12 with the amine DDM); changes in the electric modulus are related to the progress of the reaction. $\mathbf{b}$ ) changes in the maximum of M" for mixtures 1 and 2 (with the amine DDM) c) changes in the maximum of M" for mixtures 3 and 4 (with the amine DMAP) a)

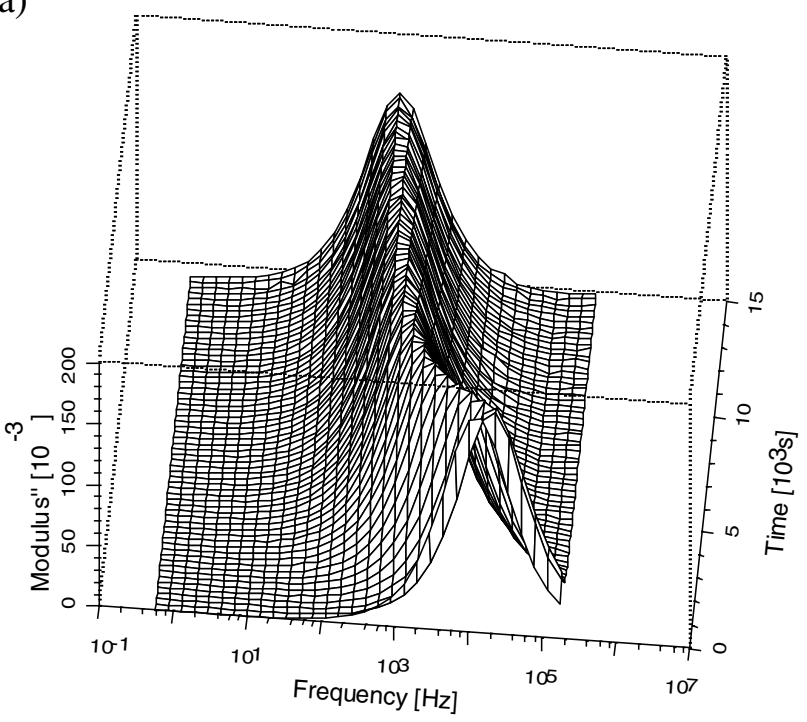

b)

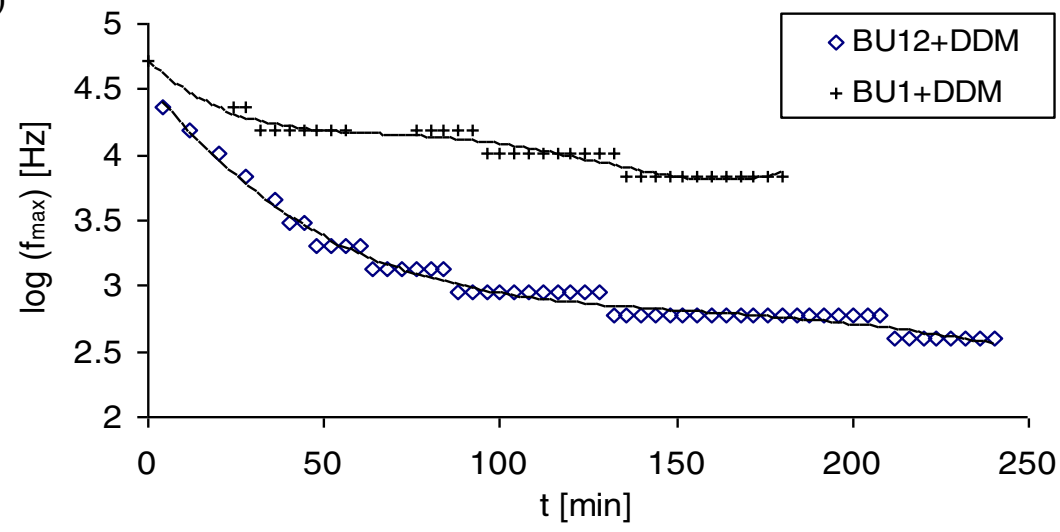

c)

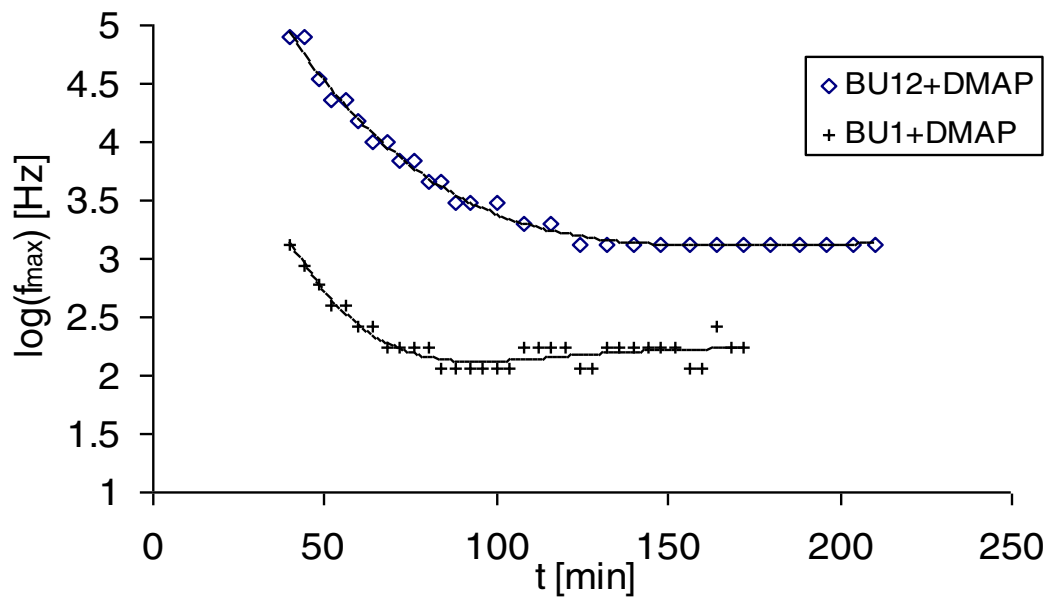

So we can conclude that the longer carbon chain had an influence on decreasing the temperature of glass transition and rising the activation energy of processes connected with the molecular mobility of dipolar groups.

For the other two products (product 3 and 4) produced from the amine DMAP, dielectric response was quite different (Fig. 5). In both the cases, only one process was detected
(Fig. 5a, b) - due to high conductivity the results were presented in the modulus representation. In product 3 the relaxation process is well seen so long as the conductivity is not too high. Example results for the temperature of $293 \mathrm{~K}$ were shown in Fig. 5c. Low temperatures at which the peak appears and its Arrhenius-like dependence on temperature (Fig. 5d) suggests it is a $\beta$-process connected with dipolar 

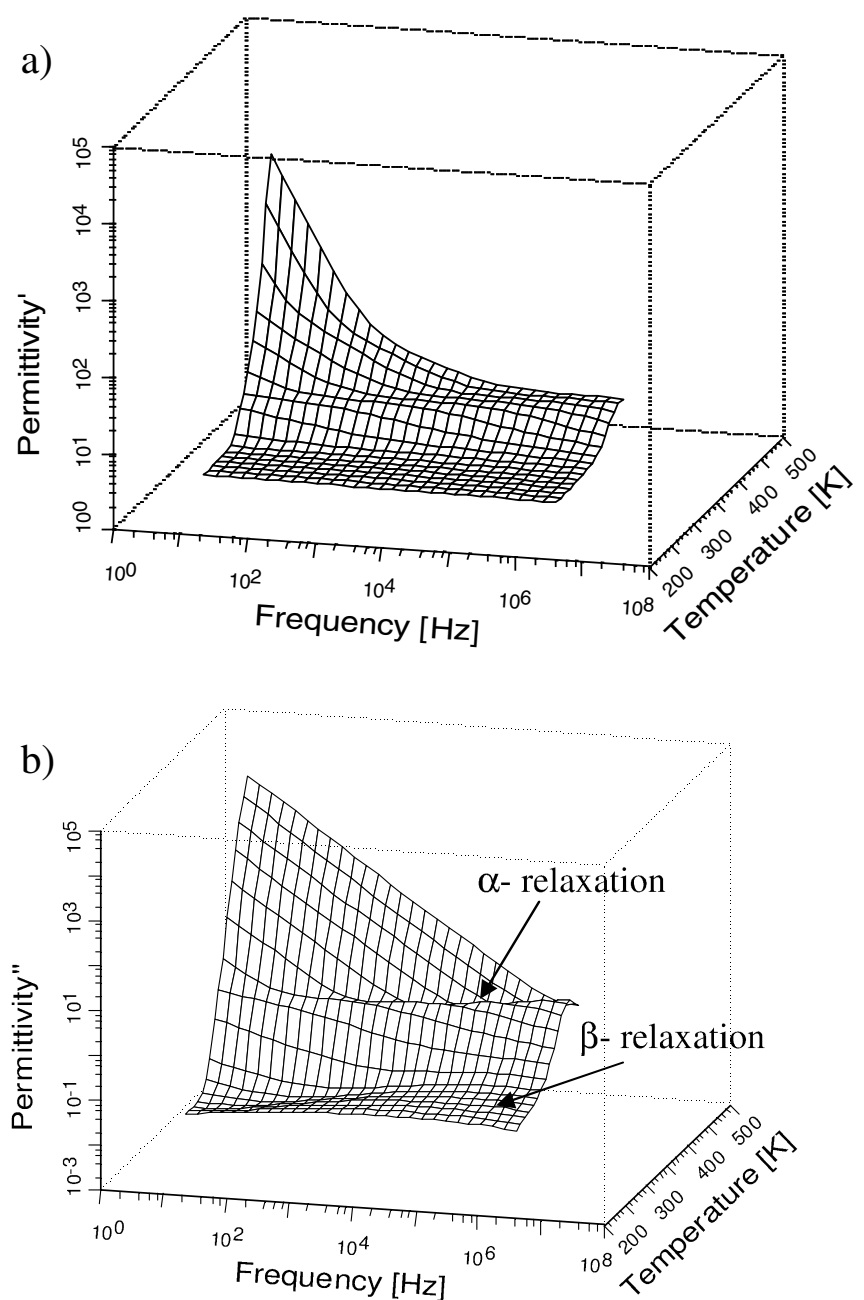

Fig. 3 Dielectric response obtained for product 1: a) a 3D representation of the real part of the electric permittivity in a wide range of frequency and temperature b) a 3D representation of the imaginary part of the electric permittivity in a wide range of frequency and temperature c) dielectric loss vs. frequency at two temperatures: (o) $303 \mathrm{~K}$ and (+) $353 \mathrm{~K}$; the solid

groups in the end of the mesogen. The activation energy obtained for this process $(0.47 \mathrm{eV})$ is similar to the one calculated for the $\beta$-process in product 1 (containing the same monomer), which suggests the same relaxation mechanism. This kind of process is also seen in the last material (product 4), but in this case the absorption maximum is shifted towards high frequency, beyond our experimental range, so it is difficult to make the full analysis (Fig. 5b).

The lack of the $\alpha$-process in the plots shown in Fig. 5a and $\mathrm{b}$ cannot unequivocally prove that such a process does not occur in products 3 and 4 at all, particularly at higher temperatures. At elevated temperatures, electric conductivity is relatively high in these materials and has large contribution to the measured electrical quantities (it is reflected e.g. by a strong peak in the M" plot), which might have masked weaker changes related to some relaxation process potentially occurring at lower frequencies.

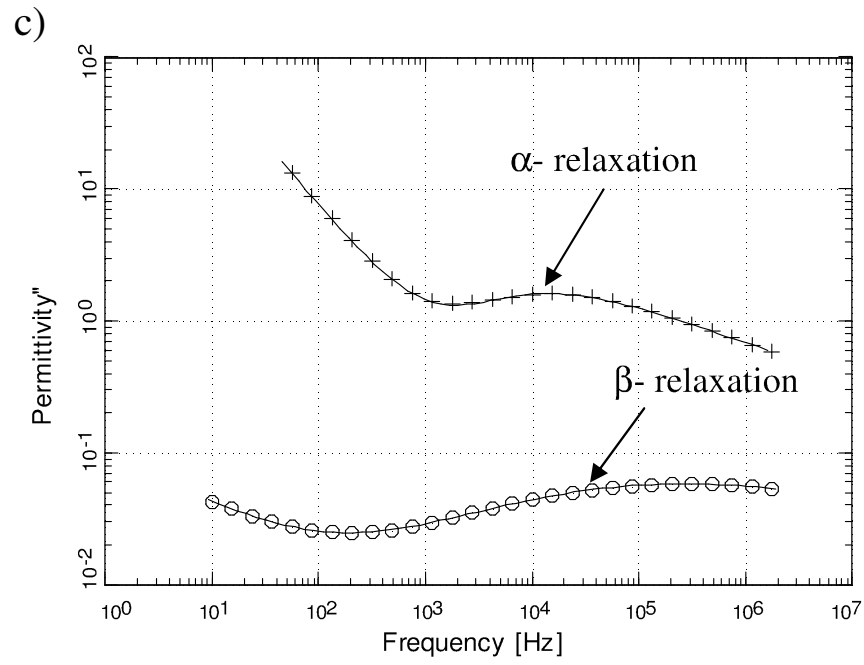

d)

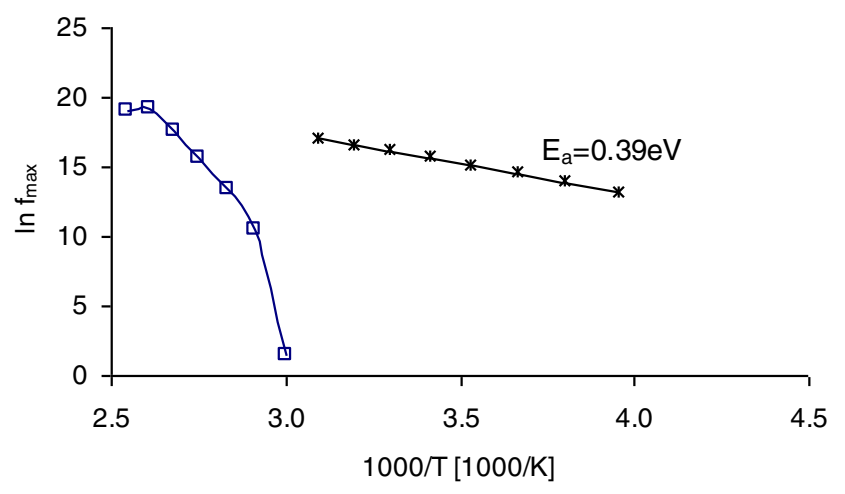

line is a fit of the Havriliak-Negami function with conductivity term to the experimental data d) relaxation peak frequency $f_{\max }$ vs. inverse temperature for both the processes; the solid lines represent fitting of the Arrhenius equation to the experimental data for the $\beta$-process

To summarise, the $\beta$-process is seen in all the products and in the material containing the longer, more flexible monomer this process is shifted towards higher frequencies and has greater activation energy.

\section{Conclusions}

This paper demonstrated the results of dielectric measurements performed on selected epoxy materials. On the grounds of optical observations, two amines were chosen as curing agents (DDM, DMAP) and optimal values of the curing temperature were established for each of the studied mixtures. The curing temperatures are somewhat higher for the monomers with longer chains.

The curing process was observed in situ by means of the dielectric spectroscopy, at previously determined temperatures. 
Fig. 4 Dielectric observations for product 2: a) a $3 \mathrm{D}$ representation of the imaginary part of the electric modulus in a wide range of frequency and temperature b) dielectric loss vs. frequency at two temperatures: $(+) 303 \mathrm{~K}$ and (o) $363 \mathrm{~K}$; the solid line is a fit of the Havriliak-Negami function with conductivity term to the experimental data c) relaxation peak frequency $f_{\max }$ vs. inverse temperature for both the processes; the solid lines represent fitting of the Arrhenius equation to the experimental data for the $\beta$-process

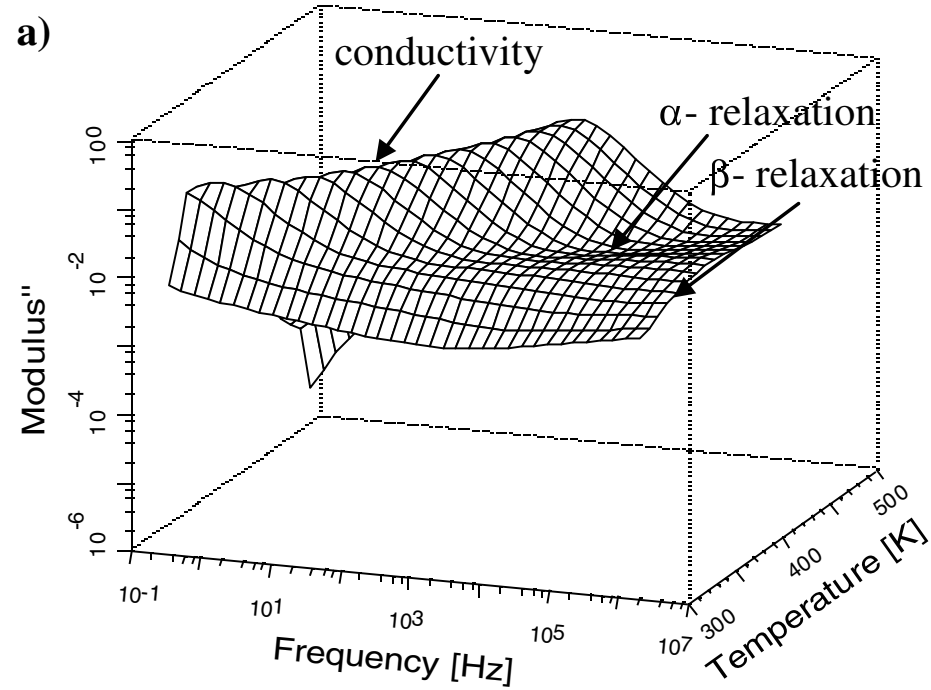

b)

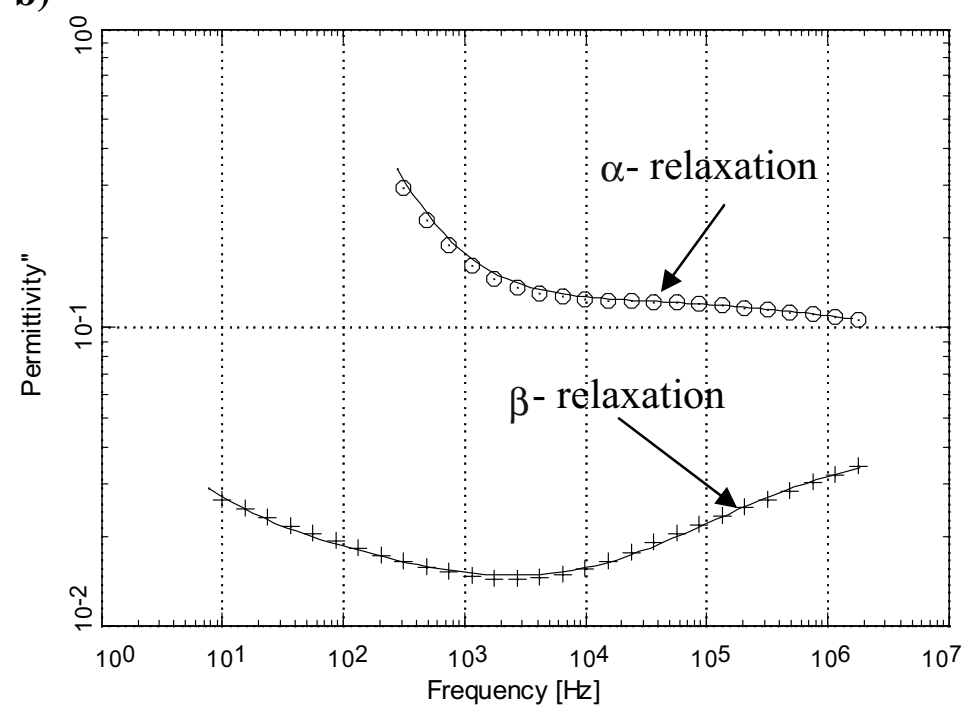

c)

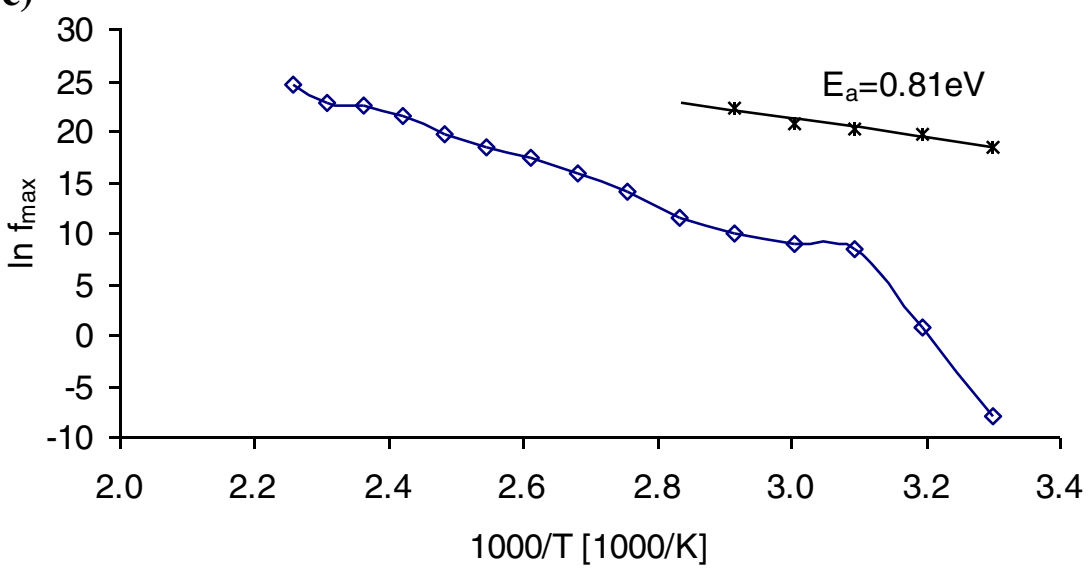

The curing reactions were observed in all the materials, but they exhibited slight differences in the reaction dynamics. The processes were seen less clearly and showed faster changes in the mixtures with shorter monomers whereas the reaction progressed more gradually in the mixtures with longer monomers.

On the basis of dielectric investigations carried out in a broad range of temperature and frequency it was concluded 


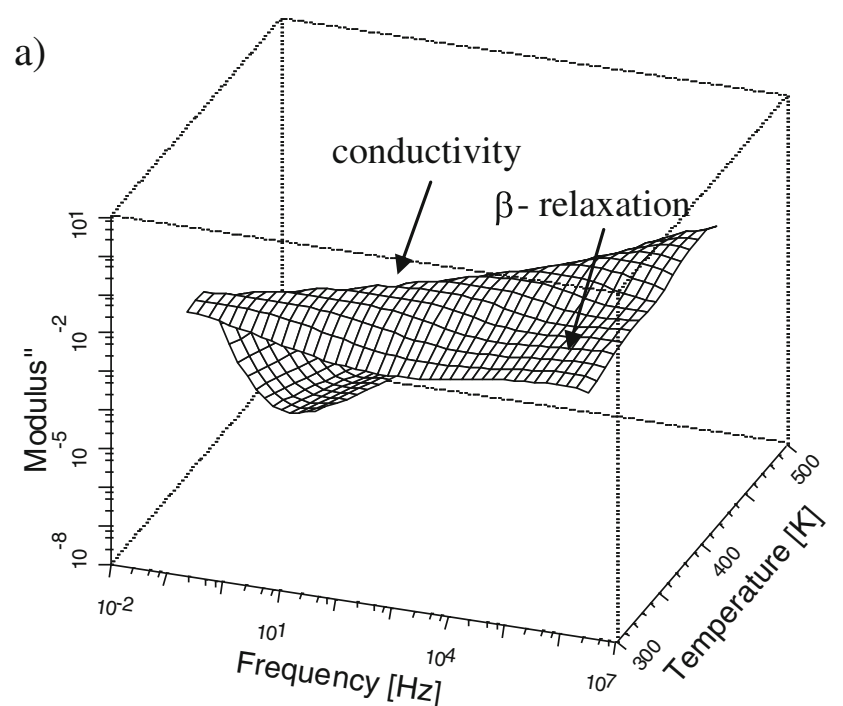

b)

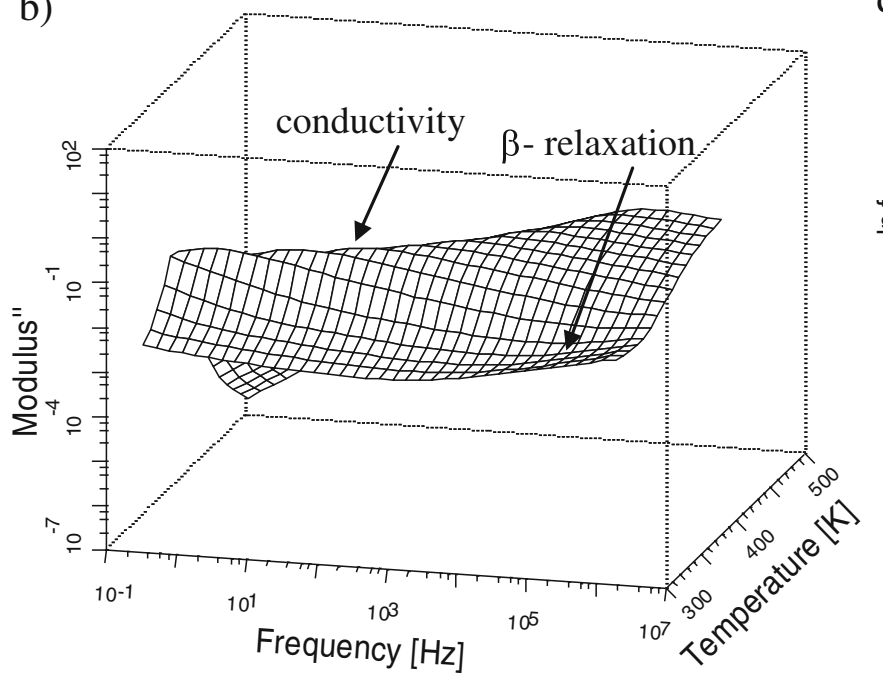

Fig. 5 Comparison of dielectric measurements for products 3 and 4 (with the amine DMAP) a) the imaginary part of the electric modulus for product $3 \mathbf{b}$ ) the imaginary part of the electric modulus for product $4 \mathbf{c}$ )

that two relaxation processes occur in the compounds with DDM as the curing agent. In the case of product 1 , the structural $\alpha$ relaxation was visible in the temperature range $253 \mathrm{~K}-323 \mathrm{~K}$, and vanishing thereof enabled estimating the vitrification temperature as $T g=333 \mathrm{~K}$. The $\beta$-process was observed at temperatures where the $\alpha$-process has already vanished (below $333 \mathrm{~K}$ ), with activation energy determined as $E a=0.39 \mathrm{eV}$. This process was visible in all the cases, which suggests that it may be connected with polar $\mathrm{COO}$ groups appearing in both the monomers. In product 2 , both the processes can be observed at temperatures between $303 \mathrm{~K}$ and $343 \mathrm{~K}$, and only the structural relaxation is present above that range. The estimated vitrification temperature, related to vanishing of the $\alpha$-process, is $303 \mathrm{~K}$. Due to simultaneous occurrence of both processes, the activation energy determined

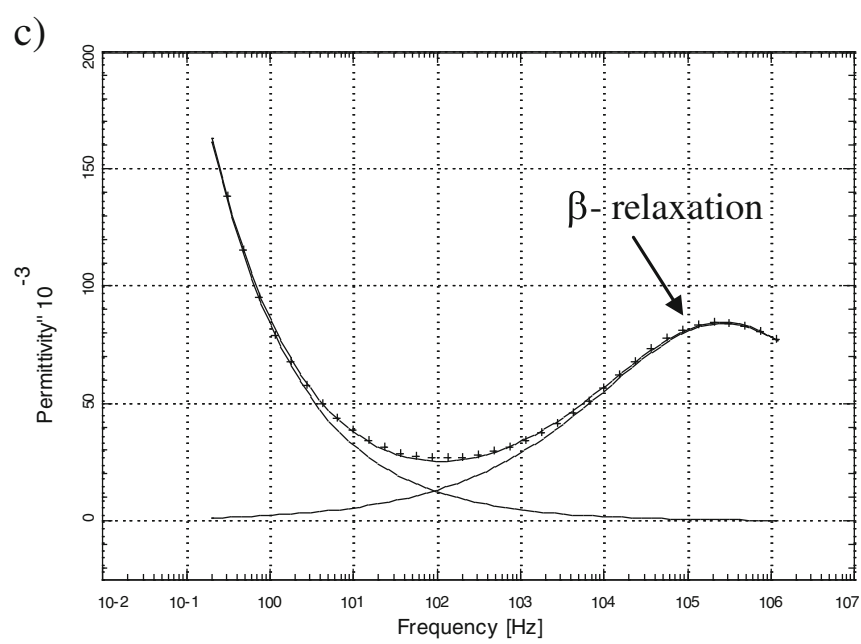

d)

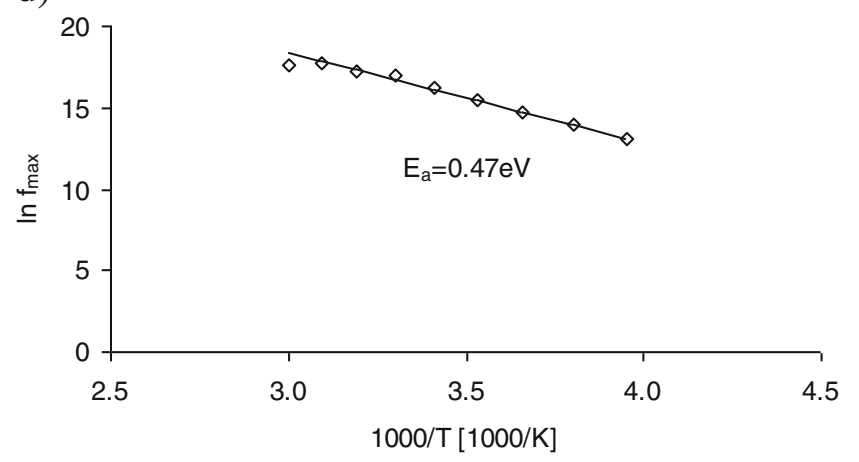

an example of fitting the Havriliak-Negami formula to the experimental data for product 3 at temperature $T=293 \mathrm{~K}$. d) the activation energy $\left(E_{a}\right)$ for the relaxation processes in product 3 for the $\beta$-process at $0.81 \mathrm{eV}$ bears a substantial amount of measurement uncertainty.

In the mixures with DMAP as the precursor, only one relaxation process was observed. It was identified as the $\beta$ process. In the case of product 3 (having shorter aliphatic chains) the process appeared at temperatures between $300 \mathrm{~K}$ and $395 \mathrm{~K}$, with activation energy estimated at $0.47 \mathrm{eV}$. Similar conclusion can be drawn from the study of product 4 . Only one relaxation occurs in this case as well, but it is difficult to describe in more detail because it is poorly visible, it could only be observed at a few temperatures around $360 \mathrm{~K}$.

Acknowledgments Part of this work received financial support from Structural Funds in the Operational Programme - Innovative Economy (IE OP) financed from the European Regional Development Fund - 
Project "Modern material technologies in aerospace industry", No. POIG.01.01.02-00-015/08-00.

Open Access This article is distributed under the terms of the Creative Commons Attribution License which permits any use, distribution, and reproduction in any medium, provided the original author(s) and the source are credited.

\section{References}

1. Urayama K, Honda S, Takigawa T (2005) Macromol 38(9):3574-3576

2. Shenoy DK, Thomsen DL, Keller P, Ratna BR (2003) J Phys Chem B 107(50):13755-13757

3. Mallon JJ, Adams PM (1993) J Polym Sci A: Polym Chem 31:22492260

4. Barclay GG, McNamee SG, Ober CK, Papathomas KI, Wang DW (1992) J Polym Sci A: Polym Chem 30:1845-1853

5. Jahromi S, Kuipers WAG, Norder B, Mijs WJ (1995) Macromol 28(7):2201-2211

6. Körner H, Shiota A, Bunning TJ, Ober CK (1996) Science 272(5259):252-255

7. Lee JY, Jang J (2007) Polym Bull 59(2):261-268

8. Mossety-Leszczak B, Włodarska M (2011) In: Iwan A, SchabBalcerzak E (eds) Liquid crystalline organic compounds and polymers as materials XXI century: from synthesis to applications. Transworld Research Network, Kerala

9. Nakka JS, Jansen KMB, Ernst LJ (2011) J Polym Res 18(6):1879-1888

10. Duan J, Kim C, Jiang P (2009) J Polym Res 16(1):45-54

11. Suzuki N, Kiba S, Yamauchi Y (2012) J Nanosci Nanotech 12:983-987

12. Suzuki N, Kiba S, Yamauchi Y (2011) J Mater Chem 21:1494114947

13. Suzuki N, Kiba S, Yamauchi Y (2011) Mater Lett 65:544-547
14. Kiba S, Okawauchi Y, Suzuki N, Yamauchi Y (2010) Chem-An Asian J 5:2100-2105

15. Vega A, Kovacs JZ, Bauhofer W, Schulte K (2009) Comp Sci Tech 69:1540-1546

16. Włodarska M, Bak G, Mossety-Leszczak B, Galina H (2009) J Mater Process Technol 209:1662-1671

17. Lee JY, Jang J (2006) Polymer 47:3036-3042

18. Castell P, Serra A, Galià M, Giamberini M, Carfagna C (2003) J Polym Sci A: Polym Chem 41:1-12

19. Cho S, Douglas EP (2002) Macromol 35(11):4550-4552

20. Hirn B, Carfagna C, Lanzetta R (1996) J Mater Chem 6(9):14731478

21. Davis FJ, Mitchell GR (1996) Polymer 37(8):1345-1351

22. Przygocki W, Włochowicz A (2001) Fizyka Polimerów. Wybrane zagadnienia. PWN, Warszawa

23. Fragiadakis D, Pissis P (2007) J Non-Cryst Solids 353(47-51):4344 4352

24. Prevosto D, Capacciolli S, Sharifi S, Kessairi K, Lucchesi M, Rolla PA (2007) J Non-Cryst Solids 353:4278-4282

25. Mohomed K (2005) Polymer 46:3847-3851

26. Rault J (2011) J Non-Cryst Solids 357:339-345

27. Rault J (2000) J Non-Cryst Solids 271:177-217

28. Rault J (1999) J Non-Cryst Solids 260:164-166

29. Kremer F, Schönhals A (2003) Broadband dielectric spectroscopy. Springer, Berlin

30. Johari GJ, Goldstein M (1970) J Chem Phys 53(6):2372-2388

31. Wlodarska M, Mossety-Leszczak B, Galina H, Bak GW, Pakula T (2004) Liq Cryst 31(4):525-534

32. Mossety-Leszczak B, Galina H, Włodarska M, Bąk GW (2006) Przemysł Chemiczny 85(8-9):956-958

33. Mossety-Leszczak B, Galina H, Włodarska M (2011) Phase Transit 84(1):15-28

34. Macedo PB, Moynihan CT, Bose R (1972) Phys Chem Glasses 13(6): 171-179

35. Havriliak S, Negami S (1967) Polymer 8:161-210 